\title{
Integrierter Finanzplan unabdingbar
}

VON ROBERT TOBIAS UND

JOHANNES WOITHON

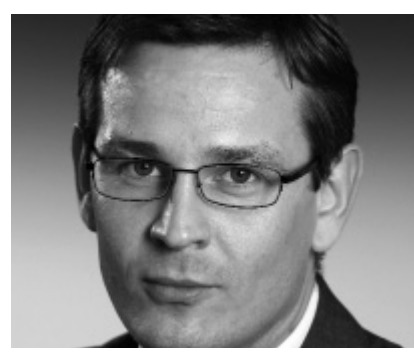

Robert Tobias ist Senior Manager der hww CMS Unternehmensberatungs $\mathrm{GmbH}$ und berät

Unternehmen bei der Restrukturierung, Sanierung und Finanzierung in Krisenzeiten. Zur hww-Gruppe gehören 360 Mitarbeiter an deutschlandweit 24 Standorten und 20 internationale Kooperationspartner. Internet http://www.cms-ag.de

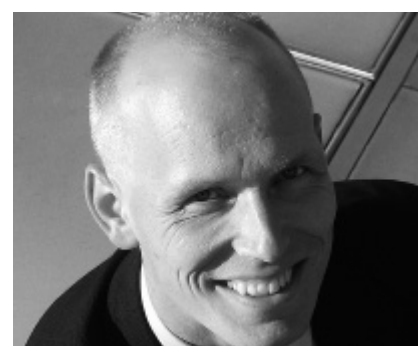

Johannes Woithon ist Geschäftsführer der Consolutions

Unternehmensberatung, die seit mehr als elf Jahren ausschließlich Unternehmen und Organisationen aus der Sozialwirtschaft berät. Zu den Tätigkeitsfeldern gehört u. a. die Strategie-,

Management- und Organisationsberatung - auch in Krisensituationen.

Internet

http://www.consolutions.de

\author{
Bei wirtschaftlichen Schwierigkeiten eines \\ Unternehmens hängt nach den neuen Bestimmungen \\ der Insolvenzordnung viel von einer begründeten \\ positiven Prognose ab: Überschuldung liegt danach \\ auch bei fehlendem Vermögen zur Deckung der \\ Verbindlichkeiten nicht vor, wenn die Fortführung des \\ Unternehmens nach den Umständen wüberwiegend \\ wahrscheinlich» ist.
}

Was für Jahrzehnte in der Sozialwirtschaft undenkbar war, ist heute bittere Realität: Wirtschaftliche Krisen sozialwirtschaftlicher Unternehmen sind an der Tagesordnung; kleine Träger sind davon genauso betroffen wie große Organisationen.

Wenn die Existenz auf dem Prüfstand steht, heißt es schnell und kompetent zu entscheiden und zu handeln. Voraussetzung dafür ist die Fähigkeit, die Situation richtig beurteilen und die möglichen Handlungsoptionen mit deren Konsequenzen genau zu kennen.

Welche Maßnahmen zu ergreifen sind, hängt dabei entscheidend von der Einschätzung $\mathrm{ab}$, inwieweit das Unternehmen auf Dauer wirtschaftlich fortbestehen kann. Diese Fragestellung ist sowohl für die Unternehmensleitung als auch die Stakeholder (beispielsweise Banken, Mitarbeiter, Lieferanten) von hoher Relevanz. In der Unternehmenskrise und Sanierungspraxis spielt diese Fragestellung seit jeher eine zentrale Rolle.

Durch das vom deutschen Gesetzgeber im Zuge der Finanzmarktkrise am 17. Oktober 2008 verabschiedete Finanzmarktstabilisierungsgesetz wurden die Voraussetzungen für den Insolvenztatbestand Überschuldung nach $\mathbb{1 9}$ (2) InsO befristet neu geregelt (gegenwärtig befristet bis 31.12.2013). Seitdem stellt die Fortbestehensprognose für Kapitalgesell- schaften sowie Unternehmen ohne Rechtspersönlichkeit, bei denen kein persönlich haftender Gesellschafter eine natürliche Person ist ( $\mathrm{GmbH} \& \mathrm{Co}$. KG), die zentrale Prüfung im Rahmen von Sanierungsprozessen dar.

Die im Januar 1999 in Kraft getretene Insolvenzordnung ( $\mathrm{Ins} \mathrm{O}$ ) benennt für Kapitalgesellschaften in den $\mathbb{S} \mathbb{S} 17$ bis 19 drei Insolvenzantragsgründe. Nach $\mathbb{} 17$ InsO liegt Zahlungsunfähigkeit vor, wenn ein Unternehmen nicht innerhalb von drei Wochen mindestens 90 Prozent seiner fälligen Verbindlichkeiten bezahlen kann. Nach $\mathbb{S} 18$ InsO kann ein Geschäftsführer oder Vorstand die Eröffnung des Insolvenzverfahrens über das Vermögen der von ihm vertretenen Gesellschaft wegen drohender Zahlungsunfähigkeit beantragen. Nach $\mathbb{} 19$ InsO liegt für juristische Personen eine Insolvenzantragspflicht vor, wenn die Gesellschaft überschuldet ist.

Vor der befristeten Neufassung des $\mathbb{S}$ 19 InsO entschied das Ergebnis der Fortbestehensprognose lediglich darüber, welcher Bewertungsansatz (Fortführung, Liquidation) im Rahmen der Überschuldungsprüfung zu wählen ist. Im Mittelpunkt standen die bilanzielle Kapitalsituation und damit der rechnerisch-materielle Überschuldungsbegriff. In der Sanierungspraxis bedeutete dies für Verhandlungen mit Gläubigern, ins- 
besondere mit Kreditinstituten, dass etwaige Insolvenzantragspflichten wegen rechnerischer Überschuldung durch Kapital stützende Maßnahmen wie beispielsweise qualifizierte Rangrücktritte beseitigt werden mussten.

Mit Art. 5 FMStG wurde die vor der Einführung der Insolvenzordnung (1999) geltende zweistufige Überschuldungsprüfung wieder eingeführt mit der Konsequenz, dass die Prüfung auf positives Fortbestehen den ersten und wichtigsten Schritt zur Vermeidung von Insolvenzantragspflichten und zur Vermeidung zivil- und strafrechtlicher Haftungsfolgen für die Geschäftsführer oder den Vorstand darstellt. Eine positive Fortbestehensprognose entlastet in diesem Sinne aber nur, sofern sie selbst belastbar ist. Für die » richtige « Erstellung einer Fortbestehensprognose gibt der Gesetzgeber indes keine Kriterien zur Hand.

\section{Kriterien einer Fortbestehensprognose}

Die Erstellung einer Fortbestehensprognose basiert auf einer Prognose der zukünftigen Zahlungsfähigkeit, üblicherweise für das laufende und das folgende Geschäftsjahr, mindestens aber für 18 Monate. Die Zahlungsfähigkeitsprognose ist zwingend auf Basis eines integrierten Finanzplans durchzuführen und in die Gesamtplanung des Unternehmens einzubinden ist. Eine isolierte Liquiditätsplanung genügt ausdrücklich nicht. Eine positive Fortbestehensprognose besteht, wenn die Zahlungsfähigkeit des Unternehmens im Prognosezeitraum mit überwiegender Wahrscheinlichkeit aufrechterhalten und nachvollziehbar begründet werden kann.

Neben der Liquiditätsperspektive und dem Prognosezeitraum kommt es somit auf die »überwiegende Wahrscheinlichkeit « an. Diese liegt beispielsweise definitiv nicht vor, wenn ein Unternehmen nach $\mathbb{1 7}$ InsO bereits heute zahlungsunfähig ist. Der Terminus »überwiegende Wahrscheinlichkeit « ist allerdings kein Maß, welches durch statistische Wahrscheinlichkeitsrechnung oder risikomathematische Szenarientechnik bestimmt werden kann. Es ist als juristisches Beweismaß aufzufassen. Die Konstatierung einer positiven Fortbestehensprognose zur Abwehr etwaiger Insolvenzantragspflichten basiert somit nicht auf unterschiedlichen Planungs- und Prognoses- zenarien, die in ihrer Mehrzahl für den Fortbestand des Unternehmens im Prognosezeitraum sprechen. Vielmehr geht es darum, die im Rahmen einer integrierten Geschäftsplanung ausgewiesene Finanz- und Liquiditätsplanung sowie die zur Realisierung erforderlichen Maßnahmen transparent darzustellen, als realistisch durchführbar zu begründen und zeitlich einzuordnen.

In der Restrukturierungs- und Sanierungspraxis hat sich infolge dessen die Verhandlungsperspektive mit den relevanten Stakeholdern (Gesellschafter, Arbeitnehmervertretung, Kreditinstitute) verschoben. Im Mittelpunkt stehen Ertrag und Liquidität steigernde Maßnahmen und weniger die bilanzielle Entlastung der Passivseite durch Rangrücktritte der Kreditgeber. Kreditinstitute werden ihren Sanierungsbeitrag in Form von Neukreditvergabe, Kreditprolongation, Kapitaldienststundung oder Sanierungszinsvereinbarung erst dann zu leisten bereit sein, wenn im und vom Unternehmen selbst kurzfristig deutliche Maßnahmen umgesetzt werden.

Beispielsweise lässt sich in der stationären Altenhilfe die Auslastung einer Einrichtung zur Ergebnisverbesserung in der Regel nur mit langfristig wirkenden und strategischen Mitteln steigern. Deshalb müssen gleichzeitig kurzfristig wirksamen Maßnahmen konsequent umgesetzt werden. Dazu zählt die Einstufung der Bewohner entsprechend ihrem tatsächlichen Pflegeaufwand. Oftmals sind dazu parallel entsprechende Schulungsmaßnah- men für die Pflegekräfte durchzuführen. Auch eine Anpassung des Personals an die tatsächliche Belegung muss ohne Verzögerung sichergestellt werden. Die Verringerung der Ausfallzeiten und der Mitarbeiter-Fluktuation ist oftmals ein weiteres entscheidendes Thema.

Eine positive Fortbestehensprognose ist ein elementarer Bestandteil der " Anforderungen an die Erstellung von Sanierungskonzepten « (IDW S6) des Instituts der Wirtschaftsprüfer e. V. Insbesondere Kreditinstitute, aber auch Arbeitnehmervertretungen und öffentliche Fördermittelgeber treffen ihre Entscheidungen heutzutage u. a. auf Basis von positiven Fortbestehensprognosen.

Belastbare Fortbestehensprognosen sind deshalb nicht nur in der Ertrags- und Liquiditätskrise ein unverzichtbares Instrument des Managements.

\section{Pflichten der Geschäftsleitung}

Durch das größere Gewicht der Fortbestehensprognose sollten Geschäftsleiter zukünftig ein besonderes Augenmerk auf die Unternehmensplanung sowie deren sorgfältige Dokumentation richten. Bereits die Rechtsprechung vor Inkrafttreten der InsO als auch die jüngere Rechtsprechung haben hervorgehoben:

- Liegt eine rechnerische Überschuldung vor, die der Gläubiger im Rahmen eines Rechtsstreits darzulegen und zu beweisen hat, obliegt der organschaftlichen Geschäftsführung die Darlegungs- sowie Beweislast für die positive Fortbestehensprognose.

- Der Geschäftsleitung kommt eine gewisse Überlegungsfrist beziehungsweise ein gewisser Beurteilungsspielraum für die Fortbestehensprognose zu.

- Für die Fortbestehensprognose kommt es nicht auf nachträgliche Erkenntnisse an, sondern allein auf die Ex-ante-Sicht eines ordentlichen Geschäftsleiters.

- Die organschaftliche Geschäftsführung trifft die Pflicht, die Lage der Gesellschaft dauernd zu beobachten und sich bei Anzeichen einer Krise durch Aufstellung eines Vermögensstatus einen Überblick über den Vermögensstand zu verschaffen. Quelle: PricewaterhouseCoopers Legal Aktiengesellschaft Rechtsanwaltsgesellschaft (PwC Legal), Internet http://www.pwclegal.de/6_7_23.html 